\title{
Single Parameter Entropy of Uncertain Variables*
}

\author{
Jiajun Liu, Liang Lin, Shuai Wu \\ College of Science, Guilin University of Technology, Guilin, China \\ Email: 2008.liujiajun@163.com
}

Received May 31, 2012; revised June 30, 2012; accepted July 7, 2012

\begin{abstract}
Uncertainty theory is a new branch of axiomatic mathematics for studying the subjective uncertainty. In uncertain theory, uncertain variable is a fundamental concept, which is used to represent imprecise quantities (unknown constants and unsharp concepts). Entropy of uncertain variable is an important concept in calculating uncertainty associated with imprecise quantities. This paper introduces the single parameter entropy of uncertain variable, and proves its several important theorems. In the framework of the single parameter entropy of uncertain variable, we can obtain the supremum of uncertainty of uncertain variable by choosing a proper $q$. The single parameter entropy of uncertain variable makes the computing of uncertainty of uncertain variable more general and flexible.
\end{abstract}

Keywords: Uncertain Distribution; Entropy of Uncertain Variable; Single Parameter Entropy

\section{Introduction}

The concept of entropy was founded by Shannon [1] in 1949 , which is a measurement of the degree of uncertainty of random variables. In 1972, De Luca and Termini [2] introduced the definition of fuzzy entropy by using Shannon function. Inspired by the Shannon entropy and fuzzy entropy, Liu [3] in 2009 proposed the concept of entropy of uncertain variable, where the entropy characterizes the uncertainty of uncertain variable resulting from information deficiency.

Tsallis Entropy initiated by Tsallis [4-6] in 1988, this is based on the following single parameter generalization of the Shannon entropy:

$$
S_{q}=k \frac{1-\sum_{i=1}^{n} p_{i}}{q-1}
$$

where $k$ is a conventional positive constant, which is usually set equal to $1, n$ is the total number of microsopic configurations, and $p_{i}$ is the set of associated probabilities $\left(\sum_{i=1}^{n} p_{i}=1\right)$. For the equiprobability distribution $p_{i}=1 / n(i=1,2, \cdots, n)$, the value of Tsallis entropy $S_{q}=\left(1-n^{1-q}\right) /(q-1)$, where $S_{q}$ is a monotonic increasing function of $n, q$ is a real number. It is clearly that in the limit $q \rightarrow 1, S_{q}$ recovers the Shannon entropy formula:

\footnotetext{
*This research was supported by the Guangxi Natural Science Foundation of China under the Grant No. 2011GXNSFA018149, Innovation Project of Guangxi Graduate Education under the Grant No. $2011105960202 \mathrm{M} 31$.
}

$$
S=-\sum_{i=1}^{n} p_{i} \ln p_{i}
$$

Henceforth, many scholars conduct to research the tsallis entropy, such as S. Abe [7], S. Abe and Y. Okamoto [8], R. J. V. dos Santos [9] and so on.

Uncertainty theory was founded by Liu [10] in 2007 and refined by Liu [11] in 2010, which is a branch of mathematics based on normality, monotonicity, selfduality, countable subadditivity, and product measure axioms. It is a effectively mathematical tool disposing of imprecise quantities in human systems. In recent years, Uncertainty theory was widely developed in many disciplines, such as uncertain process [12], uncertain calculus [3], uncertain differential equation [3], uncertain logic [13], uncertain inference [14], uncertain risk analysis [15], and uncertain statistics [11]. Meanwhile, Liu [16] proposed a spectrum of uncertain programming and applied it into system reliability design, facility location problems, vehicle routing problems, project scheduling problems and so on.

In order to provide a quantitative measurement of the degree of uncertainty in relation to an uncertain variable, Liu [3] proposed the definition of uncertain entropy resulting from information deficiency. Dai and Chen [17] investigated the properties of entropy of function of uncertain variables. The principle of maximum entropy for uncertain variables are introduced by Chen and Dai [18]. Besides, there are many literature concerning the definition of entropy of uncertain variables, such as Chen [19], Dai [20], etc. 
Inspired by the tsallis entropy, this paper introduces a new type of entropy, single parameter entropy in the framework of uncertain theory and discusses its properties. Consequently, we generalize the entropy of uncertain variable. The rest of the paper is organized as follows. In Section 2, we recall some basic concepts and theorems of uncertain theory. In Section 3, the definition of single parameter entropy of uncertain variables is proposed. In addition, some examples of the single parameter entropy are illustrated. In Section 4, several properties of single parameter entropy are proved. In Section 5, gives some discussions of single parameter entropy. In Section 6, some examples of single parameter entropy are given. At last, a brief summary is drawn.

\section{Preliminaries}

In this section, we will recall several basic concepts and theorems in the uncertain theory.

Let $\Gamma$ be a nonempty set, and $\mathcal{L}$ a $\sigma$-algebra over $\Gamma$. Each element $\Lambda \in \mathcal{L}$ is called an event. Uncertain measure $\mathcal{M}$ was introduced as a set function satisfying the following five axioms ([10]):

Axiom 1. (Normality Axiom) $\mathcal{M}\{\Gamma\}=1$ for the universal set $\Gamma$.

Axiom 2. (Monotonicity Axiom) $\mathcal{M}\left\{\Gamma_{1}\right\} \leq \mathcal{M}\left\{\Gamma_{2}\right\}$ whenever $\Lambda_{1} \subset \Lambda_{2}$.

Axiom 3. (Self-Duality Axiom) $\mathcal{M}\{\Lambda\}+\mathcal{M}\left\{\Lambda^{c}\right\}=1$ for any event $\Lambda$.

Axiom 4. (Countable Subadditivity Axiom) For every countable sequence of events $\left\{\Lambda_{i}\right\}$, we have

$$
\mathcal{M}\left\{\bigcup_{i=1}^{\infty} \Lambda_{i}\right\} \leq \sum_{i=1}^{\infty} \mathcal{M}\left\{\Lambda_{i}\right\} .
$$

Axiom 5. (Product Measure Axiom) Let $\Gamma_{k}$ be nonempty sets on which $\mathcal{M}_{k}$ are uncertain measures $k=1,2, \cdots, n$, respectively. Then the product uncertain measure $\mathcal{M}$ is an uncertain measure on the product $\sigma$-algebra $\mathcal{L}_{1} \times \mathcal{L}_{2} \times \cdots \times \mathcal{L}_{n}$ satisfying

$$
\mathcal{M}\left\{\prod_{k=1}^{n} \Lambda_{k}\right\} \leq \min _{1 \leq k \leq n} \mathcal{M}_{k}\left\{\Lambda_{k}\right\} .
$$

where $\Lambda_{k} \in \mathcal{L}_{k}, k=1,2, \cdots, n$.

We will introduce the definitions of uncertain variable and uncertainty distribution as follows.

Definition 2.1 (Liu [10]) Let $\Gamma$ be a nonempty set, and $\mathcal{P}$ be a $\sigma$-algebra over $\Gamma$, and $\mathcal{M}$ an uncertain measure. Then the triplet $(\Gamma, \mathcal{P}, \mathcal{M})$ is called an uncertainty space.

Definition 2.2 (Liu [10]) An uncertain variable is a measurable function from an uncertainty space

$(\Gamma, \mathcal{P}, \mathcal{M})$ to the set of real numbers.

Definition 2.3 (Liu [10]) The uncertainty distribution $\Phi$ of an uncertain variable $\xi$ is defined by

$$
\Phi(x)=\mathcal{M}\{\xi \leq x\} .
$$

Theorem 2.1 (Sufficient and Necessary Condition for Uncertainty distribution [21]) A function $\Phi$ is an uncertainty distribution if and only if it is an increasing function except $\Phi(x) \equiv 0$ and $\Phi(x) \equiv 1$.

Example 2.1 An uncertain variable $\xi$ is called normal if it has a normal uncertainty distribution

$$
\Phi(x)=\left(1+\exp \left(\frac{\pi(e-x)}{\sqrt{3} \sigma}\right)\right)^{-1}
$$

denoted by $\mathcal{N}(e, \sigma)$ where $e$ and $\sigma$ are real numbers with $\sigma>0$.

Then we will recall the definition of inverse uncertainty distribution as follows.

Definition 2.4 (Liu [11]) An uncertainty distribution $\Phi$ is said to be regular if its inverse function $\Phi^{-1}$ exists and is unique for each $\alpha \in(0,1)$.

Definition 2.5 (Liu [11]) Let $\xi$ be an uncertain variable with uncertainty distribution $\Phi$. Then inverse function $\Phi^{-1}$ is called the inverse uncertainty distribution of $\xi$.

Example 2.2 The inverse uncertainty distribution of normal uncertain variable $\mathcal{N}(e, \sigma)$ is

$$
\Phi^{-1}(\alpha)=e+\frac{\sqrt{3} \sigma}{\pi} \ln \frac{\alpha}{1-\alpha} .
$$

Definition 2.6 (Independence of uncertain variable Liu [10]) The uncertain variables $\xi_{1}, \xi_{2}, \cdots, \xi_{m}$ are said to be independent if

$$
\mathcal{M}\left\{\bigcap_{i=1}^{m} \xi \in B_{i}\right\}=\min _{1 \leq i \leq n} \mathcal{M}\left\{\xi_{i} \in B_{i}\right\} .
$$

for any Borel sets $B_{1}, B_{2}, \cdots, B_{m}$ of real numbers.

Example 2.3 Let $\xi$ and $\eta$ be independent normal uncertain variables $\mathcal{N}_{1}\left(e_{1}, \sigma_{1}\right)$ and $\mathcal{N}_{2}\left(e_{2}, \sigma_{2}\right)$, respectively. Then the sum $a \xi+b \eta$ is also normal uncertain variable $\mathcal{N}\left(a e_{1}+b e_{2}, a \sigma_{1}+b \sigma_{2}\right)$ for any real number $a$ and $b$.

Finally we will recall their theorems about the operational law of independent uncertain variables.

Theorem 2.2 (Liu [11]) Let $\xi_{1}, \xi_{2}, \cdots, \xi_{n}$ be independent uncertain variables with uncertainty distribution $\Phi_{1}, \Phi_{2}, \cdots, \Phi_{n}$, respectively. If $f$ be a strictly increasing with respect to $x_{1}, x_{2}, \cdots, x_{m}$ and strictly decreasing with respect to $x_{m+1}, x_{m+2}, \cdots, x_{n}$. Then

$\xi=f\left(\xi_{1}, \xi_{2}, \cdots, \xi_{n}\right)$ is an uncertain variable with inverse uncertain distribution

$$
\Psi^{-1}(\alpha)=f\left(\Phi_{1}^{-1}(\alpha), \cdots, \Phi_{m}^{-1}(\alpha), \Phi_{m+1}^{-1}(\alpha), \cdots \Phi_{n}^{-1}(\alpha)\right) .
$$

Example 2.4 Let $\xi_{1}$ and $\xi_{2}$ be independent and positive uncertain variables with uncertainty distribution $\Phi_{1}$ and $\Phi_{2}$, respectively. Then the inverse uncertainty 
distribution of the quotient $\xi_{1} / \xi_{2}$ is

$$
\Psi_{1}^{-1}(\alpha)=\frac{\Phi_{1}^{-1}(\alpha)}{\Phi_{2}^{-1}(1-\alpha)} .
$$

\section{Single Parameter Entropy}

In this section, we will introduce the definition and theorem of single parameter entropy of uncertain variable. For the purpose, we recall the entropy of uncertain variable proposed by Liu [3].

Definition 3.1 (Liu [3]) Suppose that $\xi$ is an uncertain variable with uncertainty distribution $\Phi$. Then its entropy is defined by

$$
H(\xi)=\int_{-\infty}^{+\infty} S(\Phi(x)) \mathrm{d} x
$$

where

$$
S(\Phi(x))=-\Phi(x) \ln \Phi(x)-(1-\Phi(x)) \ln (1-\Phi(x)) .
$$

We set $0 \ln 0=0$ throughout this paper. Figure 1 illustrates Definition 3.1.

Through observing Definition 3.1 and Figure 1, we find that the selection of function $S(\Phi(x))$ is very important. For an uncertain event $A$, if its incredible degree is 0 or 1 , then the incident is no uncertainty. Conversely, when this event confidence level is 0.5 , the uncertainty of the event is maximums. Therefore, the function $S(\Phi(x))$ must increases on $[0,0.5]$ and decreases on $[0.5,1]$. By the enlightenment of Tsallis entropy, we try to define the single parameter entropy of uncertain variable as follows.

Definition 3.2 Suppose that $\xi$ is an uncertain variable with uncertainty distribution $\Phi$. Then its single parameter entropy is defined by

$$
H_{q}(\xi)=\int_{-\infty}^{+\infty} S_{q}(\Phi(x)) \mathrm{d} x
$$

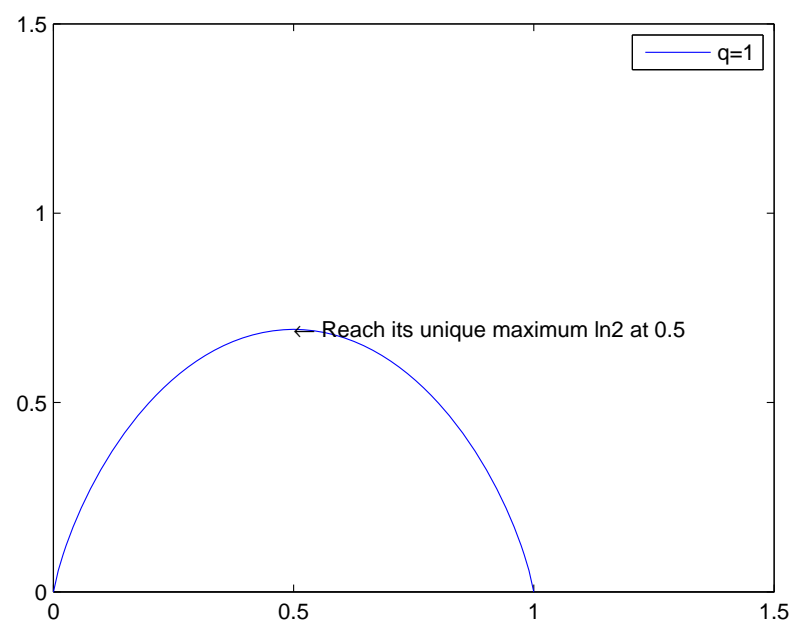

Figure 1. The entropy value of uncertain variable if and only if $q=1$. where

$$
S_{q}(\Phi(x))=\left(1-(\Phi(x))^{q}-(1-\Phi(x))^{q}\right) /(q(q-1)) .
$$

$q$ is a positive real number. For $q=1$, it is immediately verified

$$
\begin{aligned}
S_{1}(\Phi(x)) & =\lim _{q \rightarrow 1} \frac{1-(\Phi(x))^{q}-(1-\Phi(x))^{q}}{q(q-1)} \\
& =-\Phi(x) \ln \Phi(x)-(1-\Phi(x)) \ln (1-\Phi(x)) .
\end{aligned}
$$

This means that $H_{1}(\xi)$ is entropy of uncertain variable. For $q=2$, we have

$$
\begin{aligned}
S_{2}(\Phi(x)) & =\frac{1-(\Phi(x))^{2}-(1-\Phi(x))^{2}}{2(2-1)} \\
& =\Phi(x)(1-\Phi(x))
\end{aligned}
$$

It's clear that $H_{2}(\xi)$ is the quadratic entropy of uncertain variable [20]. Figure 2 illustrates Definition 3.2.

Remark 3.1 From the plot of $S_{q}(\Phi(x))$ for $\Phi(x)$ and typical values of $q$, we notice that $S_{q}(\Phi(x))$ is a monotonic function of $q$. From Definition 3.2 and the Figure 2, we can see the difference between entropy of uncertain variable and single parameter entropy, because the single parameter entropy introduces a adjustable parameter $q$, which makes the computing of uncertainty of uncertain variable more general and flexible.

Example 3.1 Let $\xi$ be an uncertain variable with uncertain distribution

$$
\Phi(x)= \begin{cases}0 & \text { if } x<a \\ 1 & \text { if } x \geq a\end{cases}
$$

Essentially, $\xi$ is constant. It follows from the definition of single parameter entropy that

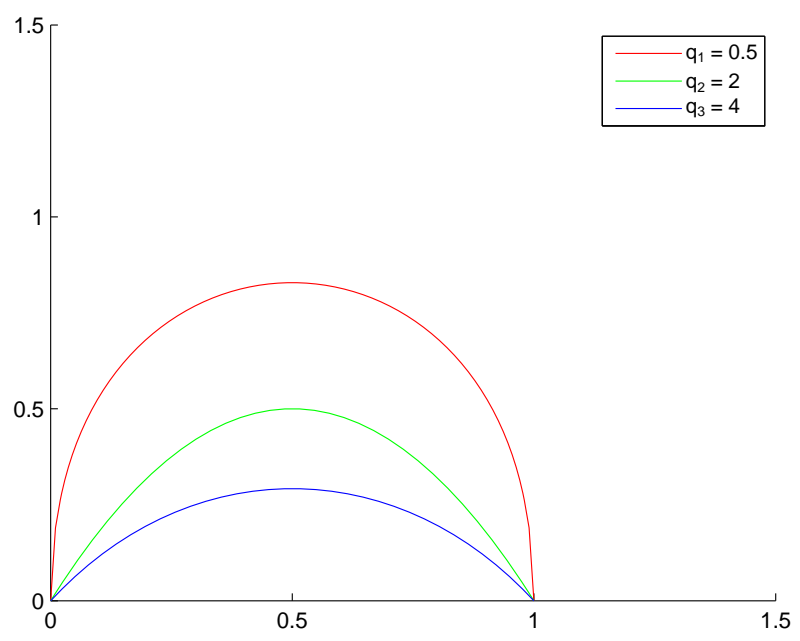

Figure 2. The different entropy value of uncertain variable with parameter $q_{1}=0.5, q_{2}=2$, and $q_{3}=4$. 


$$
\begin{aligned}
H_{q}(\xi) & =\int_{-\infty}^{a} \frac{1-0^{q}-(1-0)^{q}}{q(q-1)} \mathrm{d} x+\int_{a}^{+\infty} \frac{1-1^{q}-(1-1)^{q}}{q(q-1)} \mathrm{d} x \\
& =0
\end{aligned}
$$

This means that a constant has no uncertainty.

Example 3.2 Suppose $\xi$ be a linear uncertain variable $\mathcal{L}(a, b)$ with uncertain distribution

$$
\Phi(x)= \begin{cases}0 & \text { if } x<a \\ \frac{x-a}{b-a} & \text { if } a \leq x<b \\ 1 & \text { if } x \geq b\end{cases}
$$

Then its single parameter entropy is

$$
\begin{aligned}
H_{q}(\xi) & =\int_{a}^{b} \frac{1-\left(\frac{x-a}{b-a}\right)^{q}-\left(1-\frac{x-a}{b-a}\right)^{q}}{q(q-1)} \mathrm{d} x \\
& =\frac{b-a}{q(q+1)}
\end{aligned}
$$

especially, $H_{1}(\xi)=(b-a) / 2, H_{2}(\xi)=(b-a) / 6$.

Example 3.3 Suppose $\xi$ be a zigzag uncertain variable $\mathcal{Z}(a, b, c)$ with uncertain distribution

$$
\Phi(x)= \begin{cases}0 & \text { if } x<a \\ \frac{x-a}{2(b-a)} & \text { if } a \leq x<b \\ \frac{x+c-2 b}{2(c-b)} & \text { if } b \leq x<c \\ 1 & \text { if } x \geq c\end{cases}
$$

Then its single parameter entropy is

$$
\begin{aligned}
H_{q}(\xi) & =\int_{a}^{b} \frac{1-\left(\frac{x-a}{2(b-a)}\right)^{q}-\left(1-\frac{x-a}{2(b-a)}\right)^{q}}{q(q-1)} \mathrm{d} x \\
& +\int_{a}^{b} \frac{1-\left(\frac{x+c-2 b}{2(c-b)}\right)^{q}-\left(1-\frac{x+c-2 b}{2(c-b)}\right)^{q}}{q(q-1)} \mathrm{d} x \\
& =\frac{c-a}{q(q+1)}
\end{aligned}
$$

especially, $H_{1}(\xi)=(c-a) / 2, H_{2}(\xi)=(c-a) / 6$.

\section{Properties of Single Parameter Entropy}

Assuming the uncertain variable with regular distribution, we obtain some theorems of single parameter entropy as follows.

Theorem 4.1 Let $\xi$ is an uncertain variable. Then the single parameter entropy

$$
H_{q}(\xi) \geq 0
$$

where the equality holds if $\xi$ is a constant.

Proof: From Figure 2, the theorem is clear. As an uncertain variable tends to a constant, the single parameter entropy tends to the minimum 0 .

Theorem 4.2 Let $\xi$ be an uncertain variable, and $\$ c \$$ a real number. Then

$$
H_{q}(\xi+c)=H_{q}(\xi)
$$

that is, the single parameter entropy is invariant under arbitrary translations.

Proof: Write the uncertainty distribution of $\xi$ as $\Phi$, then

$$
\Phi(x)=\mathcal{M}\{\xi \leq x\}
$$

From this equation, we get the uncertainty distribution of uncertain variable as follow:

$$
\mathcal{M}\{\xi+c \leq x\}=\mathcal{M}\{\xi \leq x-c\}=\Phi(x-c)
$$

Using the definition of the single parameter entropy, we find

$$
\begin{aligned}
H_{q}(\xi+c) & =\int_{-\infty}^{+\infty} S_{q}(\Phi(x-c)) \mathrm{d} x \\
& =\int_{-\infty}^{+\infty} \frac{1-(\Phi(x-c))^{q}-(1-\Phi(x-c))^{q}}{q(q-1)} \mathrm{d} x \\
& =H_{q}(\xi)
\end{aligned}
$$

The theorem is proved.

Theorem 4.3 Let $\xi$ be an uncertain variable, and let $k$ be a real number, then

$$
H_{q}(k \xi)=|k| H_{q}(\xi)
$$

Proof: Denote the uncertain distribution function of $\xi$ by $\Phi$. If $k \neq 0$, then the uncertain variable $k \xi$ has an uncertain distribution function $\Phi(x / k)$. It follows from the definition of single parameter entropy that

$$
\begin{aligned}
H_{q}(k \xi) & =\int_{-\infty}^{+\infty} S_{q}\left(\Phi\left(\frac{x}{k}\right)\right) \mathrm{d} x \\
& =|k| \int_{-\infty}^{+\infty} S_{q}(\Phi(x)) \mathrm{d} x \\
& =|k| H_{q}(\xi)
\end{aligned}
$$

when $k=0$, we have $H_{q}(k \xi)=|k| H_{q}(\xi)=0$.

Theorem 4.4 Let $\xi$ be an uncertain variable with uncertain distribution $\Phi$, then

$$
H_{q}(\xi)=-\int_{0}^{1} \Phi^{-1}(\alpha) S_{q}^{\prime}(\alpha) \mathrm{d} \alpha
$$

where

$$
S_{q}^{\prime}(\alpha)=\left((1-\alpha)^{q-1}-\alpha^{q-1}\right) /(q-1),
$$

especially, 


$$
S_{1}^{\prime}(\alpha)=\ln ((1-\alpha) / \alpha), S_{2}^{\prime}(\alpha)=1-2 \alpha
$$

Proof: It is obvious that $S_{q}(\alpha)$ is a derivable function with

$$
S_{q}^{\prime}(\alpha)=\frac{(1-\alpha)^{q-1}-\alpha^{q-1}}{q-1}
$$

Since

$$
S_{q}(\Phi(x))=\int_{0}^{\Phi(x)} S_{q}^{\prime}(\alpha) \mathrm{d} \alpha=-\int_{\Phi(x)}^{1} S_{q}^{\prime}(\alpha) \mathrm{d} \alpha
$$

and noting that the uncertain variable $\xi$ has a regular uncertain distribution $\Phi$, we have

$$
\begin{aligned}
H_{q}(\xi) & =\int_{-\infty}^{+\infty} S_{q}(\Phi(x)) \mathrm{d} x \\
& =\int_{-\infty}^{+\infty} \int_{0}^{\Phi(x)} S_{q}^{\prime}(\alpha) \mathrm{d} \alpha \mathrm{d} x \\
& =\int_{-\infty}^{0} \int_{0}^{\Phi(x)} S_{q}^{\prime}(\alpha) \mathrm{d} \alpha \mathrm{d} x+\int_{0}^{+\infty} \int_{0}^{\Phi(x)} S_{q}^{\prime}(\alpha) \mathrm{d} \alpha \mathrm{d} x \\
& =\int_{-\infty}^{0} \int_{0}^{\Phi(x)} S_{q}^{\prime}(\alpha) \mathrm{d} \alpha \mathrm{d} x-\int_{0}^{+\infty} \int_{\Phi(x)}^{0} S_{q}^{\prime}(\alpha) \mathrm{d} \alpha \mathrm{d} x
\end{aligned}
$$

By Fubini theorem, we have

$$
\begin{aligned}
H_{q}(\xi) & =\int_{0}^{\Phi(0)} \int_{\Phi^{-1}(a)}^{0} S_{q}^{\prime}(\alpha) \mathrm{d} x \mathrm{~d} \alpha-\int_{\Phi(0)}^{1} \int_{0}^{\Phi^{-1}(a)} S_{q}^{\prime}(\alpha) \mathrm{d} x \mathrm{~d} a \\
& =-\int_{0}^{\Phi(0)} \int_{0}^{\Phi^{-1}(a)} S_{q}^{\prime}(\alpha) \mathrm{d} x \mathrm{~d} \alpha-\int_{\Phi(0)}^{1} \int_{0}^{\Phi^{-1}(a)} S_{q}^{\prime}(\alpha) \mathrm{d} x \mathrm{~d} a \\
& =-\int_{0}^{1} \Phi^{-1}(a) S_{q}^{\prime}(\alpha) \mathrm{d} \alpha
\end{aligned}
$$

The theorem is proved.

Theorem 4.5 Let $\xi$ and $\eta$ be independent uncertain variables, then for any real numbers $a$ and $b$, we have

$$
H_{q}(a \xi+b \eta)=|a| H_{q}(\xi)+|b| H_{q}(\eta)
$$

Proof: Suppose that $\xi$ and $\eta$ have uncertainty distribution $\Phi$ and $\Psi$, respectively, and inverse uncertainty distribution $\Phi^{-1}$ and $\Psi^{-1}$, respectively. Note that the inverse uncertainty distribution of $\xi+\eta$ is

$$
\Upsilon^{-1}(\alpha)=\Phi^{-1}(\alpha)+\Psi^{-1}(\alpha)
$$

From Theorem 4.4, we have

$$
H_{q}(\xi+\eta)=-\int_{0}^{1}\left(\Phi^{-1}(\alpha)+\Psi^{-1}(\alpha)\right) S_{q}^{\prime}(\alpha) \mathrm{d} a
$$

Since, Theorem 4.3, we obtain

$$
H_{q}(a \xi+b \eta)=|a| H_{q}(\xi)+|b| H_{q}(\eta)
$$

The theorem is proved.

Theorem 4.6 (Alternating Monotone function) Let $\xi_{1}, \xi_{2}, \cdots, \xi_{n}$ be independent uncertain variables with uncertainty distribution $\Phi_{1}, \Phi_{2}, \cdots, \Phi_{n}$, respectively. If the function $\$ \mathrm{f} \$$ is a strictly increasing with respect to $x_{1}, x_{2}, \cdots, x_{m}$ and strictly decreasing with respect to $x_{m+1}, x_{m+2}, \cdots, x_{n}$, then $\xi=f\left(\xi_{1}, \xi_{2}, \cdots, \xi_{n}\right)$ has a single parameter entropy

$$
H_{q}(\xi)=-\int_{0}^{1} \Psi^{-1}(\alpha) S_{q}^{\prime}(\alpha) \mathrm{d} \alpha
$$

where

$$
\begin{aligned}
& \Psi^{-1}(\alpha) \\
= & f\left(\Phi_{1}^{-1}(\alpha), \cdots, \Phi_{m}^{-1}(\alpha), \Phi_{m+1}^{-1}(1-\alpha), \cdots \Phi_{n}^{-1}(1-\alpha)\right) .
\end{aligned}
$$

Proof: Let $\Psi$ be the uncertainty distribution function of $f(\xi)$, then it follows from Theorem 2.2 that

$$
\begin{aligned}
& \Psi^{-1}(\alpha) \\
& =f\left(\Phi_{1}^{-1}(\alpha), \cdots, \Phi_{m}^{-1}(\alpha), \Phi_{m+1}^{-1}(1-\alpha), \cdots \Phi_{n}^{-1}(1-\alpha)\right)
\end{aligned}
$$

Since, Theorem 4.4, we have

$$
H_{q}(\xi)=-\int_{0}^{1} \Psi^{-1}(\alpha) S_{q}^{\prime}(\alpha) \mathrm{d} \alpha
$$

The theorem is proved.

Example 4.1 Let $\xi_{1}$ and $\xi_{2}$ be independent uncertain variables with regular uncertainty distribution $\Phi_{1}$ and $\Phi_{2}$, respectively. Since the function $f\left(x_{1}, x_{2}\right)=x_{1}-x_{2}$ is strictly increasing with respect to $x_{1}$ and strictly decreasing with respect to $x_{2}$. From the Theorem 2.2, the inverse uncertainty distribution of the function $\xi_{1}-\xi_{2}$ is as follow

$$
\Psi^{-1}(\alpha)=\Phi_{1}^{-1}(\alpha)-\Phi_{2}^{-1}(1-\alpha)
$$

therefore, its single parameter entropy is

$$
\begin{aligned}
H_{q}(\xi) & =-\int_{0}^{1} \Psi^{-1}(\alpha) S_{q}^{\prime}(\alpha) \mathrm{d} \alpha \\
& =-\int_{0}^{1}\left(\Phi_{1}^{-1}(\alpha)-\Phi_{2}^{-1}(1-\alpha)\right) S_{q}^{\prime}(\alpha) \mathrm{d} \alpha .
\end{aligned}
$$

\section{Discussions of Single Parameter Entropy}

Theorem 5.1 Let $\xi$ be a uncertain variable with uncertain distribution $\Phi(x)$, then

$$
H_{q}(\xi) \leq \int_{-\infty}^{+\infty} \frac{1-2^{1-q}}{q(q-1)} \mathrm{d} x
$$

where the equality holds if uncertain distribution $(\Phi(x))^{q}=(1-\Phi(x))^{q}$.

Proof: Let $\xi$ be a uncertain variable with uncertain distribution $\Phi(x), \Phi(x) \in[0,1]$, then

$$
(\Phi(x))^{q}+(1-\Phi(x))^{q} \geq 2(\Phi(x)(1-\Phi(x)))^{\frac{q}{2}}
$$

where the equality holds if $(\Phi(x))^{q}=(1-\Phi(x))^{q}$, that is $\Phi(x)=1 / 2$. Then

$$
H_{q}(\xi) \leq \int_{-\infty}^{+\infty} \frac{1-2^{1-q}}{q(q-1)} \mathrm{d} x
$$

We complete the proof.

In according to Theorem 5.1, we obtain three situa- 
tions as follows.

Situation 5.1 If uncertain variable $\xi$ is a constant $c$, that is $\xi=c$, then

$$
H_{q}(\xi) \leq 0
$$

from Theorem 4.1, we get $H_{q}(\xi)=0$ since the constant is no uncertainty.

Situation 5.2 Let uncertain variable $\xi \in[a, b]$, then

$$
H_{q}(\xi) \leq \frac{1-2^{1-q}}{q(q-1)}(b-a)
$$

According to the fact, we can find the appropriate $q$ to describe the uncertainty of uncertain variable. Especially, when $q \rightarrow 1, H_{1}(\xi) \leq \sqrt{2}(b-a)$, as $q \rightarrow 2, H_{2}(\xi) \leq(b-a) / 4$. That is, the single parameter entropy measures the uncertainty of uncertain variable more flexible than the entropy of uncertain variable.

Situation 5.3 Suppose uncertain variable $\xi$ is an impossible event. If we choose $q \rightarrow \infty$, we have

$$
H_{q}(\xi) \leq 0
$$

from Theorem 4.1, we get $H_{q}(\xi)=0$.

It is consistent with the reality, which the impossible event can be interpreted that it has no uncertainty.

\section{Example of Single Parameter Entropy}

Example 6.1 Let uncertain variable $\xi, \eta \in[a, b]$, then

$$
\begin{aligned}
& H_{q_{1}}(\xi) \leq \frac{1-2^{1-q_{1}}}{q_{1}\left(q_{1}-1\right)}(b-a) \\
& H_{q_{2}}(\xi) \leq \frac{1-2^{1-q_{2}}}{q_{2}\left(q_{2}-1\right)}(b-a)
\end{aligned}
$$

By the expert's experimental data or people's subjective judgment, we can choose a appropriate $q$ to judge the relation of $H_{q_{1}}(\xi)$ and $H_{q_{2}}(\xi)$. Furthermore, we can obtain the relation of $\xi$ and $\eta$. For instance, if two persons' age $\xi, \eta \in[20,30]$ and they are about 25 years old, Suppose we obtain $q_{1}=0.5, q_{2}=3$, then $H_{q_{1}}=16.56, H_{q_{2}}=1.25$. It is clear that $\eta$ is more close to 25 years old than $\xi$.

For some case, the entropy of uncertain variable is invalid. However, the single parameter entropy of uncertain variable works well. The follow example shows the point.

Example 6.2 Assume that the uncertain variable $\xi$ has uncertain distribution as follow

$$
\Phi(x)= \begin{cases}1-\frac{1}{x} & \text { if } x \geq 1 \\ 0 & \text { if } x<1\end{cases}
$$

we get the entropy of uncertain variable as follow:

$$
\begin{aligned}
H_{q}(\xi) & =\int_{1}^{+\infty}-\Phi(x) \ln \Phi(x)-(1-\Phi(x)) \ln (1-\Phi(x)) \mathrm{d} x \\
& \geq \int_{1}^{+\infty}-\frac{1}{x} \ln \frac{1}{x} \mathrm{~d} x \\
& =\infty
\end{aligned}
$$

It is clear that entropy of uncertain variable is infinite.

So we consider the single parameter entropy of uncertain variable.

$$
H_{q}(\xi) \leq \int_{1}^{\Phi^{-1}}\left(\frac{1}{2}\right) \frac{1-2^{1-q}}{q(q-1)} \mathrm{d} x=\frac{1-2^{1-q}}{q(q-1)}
$$

The example illustrate that we can obtain the supremum of uncertainty of uncertain variable by choosing a proper $q$. So the application of single parameter entropy is more extensive.

\section{Conclusion}

In this paper, we recalled the entropy of uncertain variable and its properties. On the basis of the entropy of uncertain variable, and inspired by the tsallis entropy, we introduce the single parameter entropy of uncertain variable and explored its several important properties. We have generalized entropy of uncertain variable because of the singe parameter entropy of uncertain variable, which makes the calculating of uncertainty of uncertain variable more general and flexible by choosing an appropriate $q$.

\section{REFERENCES}

[1] C. Shannon, "The Mathematical Theory of Communication," The University of Illinois Press, Urbana, 1949.

[2] A. De Luca and S. Termini, "A Definition of Nonprobabilitistic Entropy in the Setting of Fuzzy Sets Theory," Information and Control, Vol. 20, 1972, pp. 301-312.

[3] B. Liu, "Some Research Problems in Uncertainty Theory," Journal of Uncertain Systems, Vol. 3, No. 1, 2009, pp. 3-10.

[4] A comprehensive list of references can currently be obtained from http://tsallis.cat.cbpf.br/biblio.htm

[5] C. Tsallis, "Possible Generalization of Boltzmann-Gibbs," Statistics, Vol. 52, No. 1-2, 1988, pp. 479-487. doi:10.1007/BF01016429

[6] C. Tsallis, "Non-Extensive Thermostatistics: Brief Review and Comments," Physica A, Vol. 221, No. 1-3, 1995, pp. 277-290. doi:10.1016/0378-4371(95)00236-Z

[7] S. Abe, "Axiom and Uniqueness Theorem for Tsallis Entropy," Physics Letters A, Vol. 271, No. 1-2, 2000, pp. 74-79. doi:10.1016/S0375-9601(00)00337-6

[8] S. Abe and Y. Okamoto, "Nonextensive Statistical Mechanics and Its Applications, Lecture Notes in Physics," Springer-Verlag, Heidelberg, 2001. doi:10.1007/3-540-40919-X 
[9] R. J. V. dos Santos, "Generalization of Shannon's Theorem for Tsallis Entropy," Journal of Mathematical Physics, Vol. 38, No. 8, 1997, pp. 4104-4107. doi: $10.1063 / 1.532107$

[10] B. Liu, "Uncertainty Theory," 2nd Edition, SpringerVerlag, Berlin, 2007.

[11] B. Liu, "Uncertainty Theory: A Branch of Mathematics for Modeling Human Uncertainty," Springer-Verlag, Berlin, 2010. doi:10.1007/978-3-642-13959-8

[12] B. Liu, "Fuzzy Process, Hybrid Process and Uncertain Process," Journal of Uncertain Systems, Vol. 2, No. 1, 2008, pp. 3-16. http://orsc.edu.cn/process/071010.pdf

[13] X. Li and B. Liu, "Hybrid Logic and Uncertain Logic," Journal of Uncertain Systems, Vol. 3, No. 2, 2009, pp. 83-94.

[14] B. Liu, "Uncertain Set Theory and Uncertain Inference Rule with Application to Uncertain Control," Journal of Uncertain Systems, Vol. 4, No. 2, 2010, pp. 83-98.

[15] B. Liu, "Uncertain Risk Analysis and Uncertain Reliability Analysis," Journal of Uncertain Systems, Vol. 4, No.
3, 2010, pp. 163-170.

[16] B. Liu, "Theory and Practice of Uncertain Programming," 2nd Edition, Springer-Verlag, Berlin, 2009. doi:10.1007/978-3-540-89484-1

[17] W. Dai and X. Chen, "Entropy of Function of Uncertain Variables," Technical Report, 2009. http://orsc.edu.cn/online/090805.pdf

[18] X. Chen and W. Dai, "Maximum Entropy Principle for Uncertain Variables,” Technical Report, 2009. http://orsc.edu.cn/online/090618.pdf

[19] X. Chen, "Cross-Entropy of Uncertain Variables and Its Applications," Technical Report, 2009. http://orsc.edu.cn/online/091021.pdf

[20] W. Dai, "Maximum Entropy Principle of Quadratic Entropy of Uncertain Variables," Technical Report, 2010. http://orsc.edu.cn/online/100314.pdf

[21] Z. X. Peng and K. Iwamura, "A Sufficient and Necessary Condition of Uncertainty Distribution," Journal of Interdisciplinary Mathematics, Vol. 13, No. 3, 2010, pp. 277 285. 